\title{
Anomaly in photoelectron angular distributions
}

\author{
Jingtao Zhang, ${ }^{1}$ L. D. van Woerkom, ${ }^{2}$ Dong-Sheng Guo, ${ }^{2,3}$ and R. R. Freeman ${ }^{2}$ \\ ${ }^{1}$ State Key Laboratory for High Field Laser Physics, Shanghai Institute of Optics and Fine Mechanics, \\ Chinese Academy of Sciences, China \\ ${ }^{2}$ Department of Physics, Ohio State University, Columbus, Ohio 43210, USA \\ ${ }^{3}$ Department of Physics, Southern University, Baton Rouge, Louisiana 70813, USA
}

(Received 15 September 2006; published 27 July 2007)

\begin{abstract}
The recently observed anomaly in photoelectron angular distributions (PADs), the disappearance of the main lobes of PADs which should be usually in the direction of laser polarization, is reinterpreted as a minimum of generalized Bessel functions in the laser-polarization direction with the theory of nonperturbative quantum electrodynamics. The reinterpretation has no artificial fitting parameters and explains more features of the experimentally observed PADs, in contrast to the existing interpretation in which the anomaly is interpreted as a quantum interference of angular momentum partial waves. Some hierarchy anomalies are predicted for further experimental observations.
\end{abstract}

DOI: 10.1103/PhysRevA.76.015401

PACS number(s): 32.80.Rm, 03.65.Nk, 12.20.Ds, 42.65.Ky

The study of excess photon detachment (EPD) has unique advantages in the studies of the photoelectric effects in strong laser fields due to the vanishing ionic Coulomb effects on the final electron states $[1,2]$. In a recent experiment, Reichle, Helm, and Kiyan [3] observed an interesting anomalous phenomena in a photoelectron angular distribution (PAD) from photodetachment of $\mathrm{H}^{-}$in a strong infrared laser field where the main lobes of the PAD looked dislocated. The main emission direction of photoelectrons was not in the polarization direction of laser light as it should be in most cases, rather in the directions perpendicular to the laser polarization. Their theoretical interpretation to this observation was that a quantum interference effect due to the angularmomentum partial-wave mixing. Their parameter fitting, based on the Legendre polynomials, looked generally good, but with one artificial fitting parameter $[4,5]$.

The study of PADs using laser light has a many-decade history. The interpretations using different theories not only facilitate the understandings of the basic physics behind the experimental data, but also provide direct justifications to the interpreting theories.

Parameter fitting to PADs with partial waves has also a long history, and has been extensively used by both theoreticians and experimentalists. On the theoretical side, due to lack of knowledge of exact wave functions, the partial-wave expansion was thought as an effective method in traditional scattering theories and appeared in many books [6]. There were many theoretical studies on angular distributions especially for all kinds of photoeffects. As early as in 1948, Yang [7] proved that the angular distribution of an emitted electron from a target interacting with an incident photon in the dipole approximations was proportional to a linear combination of 1 and $\cos ^{2} \theta$ where $\theta$ was the ejection angle of the outgoing particle with respect to polarization vector of the incident photon. Later, Cooper and Zare [8] used $1+\beta P_{2}(\cos \theta)$ to express the PADs where $P_{2}(x)$ is the second-order Legendre polynomial and $\beta$ is the asymmetry parameter. Since $P_{2}(\cos \theta)=3\left(\cos ^{2} \theta-1\right) / 2$ and $P_{0}(\cos \theta)$ $=1$, Yang's result can be thought of as an early treatment to PADs using-partial wave mixing between an $s$ state and a $d$ state. Earlier theories also showed that the coefficient $\beta$ could be analytically determined [9]. Kim, Widmayer, and Girardeau [10], using hypergeometric functions, derived closed expressions for partial-wave PADs. On the experimental side, data fitting to PADs with partial waves expressed by trigonometrical functions or Legendre functions is a common technique in data analysis. Bashkansky, Bucksbaum, and Schumacher used Fourier series to fit PADs produced by elliptically polarized laser light [11]. Partial-wave decomposition was used not only in analyzing PADs, but also in analyzing total rates of the ionization due to the Freeman resonances [12]. Hansch, Walker, and Van Woerkom [13] used the partial-wave method to explain their measured photoelectrons from $f$ and $g$ low-lying Rydberg states.

The development of nonperturbative quantum electrodynamics (NPQED) made it possible to use exact wave functions such as quantum-field Volkov wave functions and nonperturbative scattering theory $[14,15]$ to treat multiphoton effects. In the frame of NPQED, we developed a theoretical approach to PADs $[16,17]$. Our theory successfully explained recent measurements on PADs [18] which showed jets sticking out from the waist of the two main lobes. That the amplitude of a photonic transition of an electron can be expressed by a generalized Bessel function proved by Reiss [19] is a common feature for nonperturbative theories. Our conclusion was that a jet signifies a maximum of a generalized Bessel function. We found the number rule to determine the number of jets: the number of jets on one side is 2 times the number of maxima of the generalized Bessel function in the domain $\left[0,\left|z_{f}\right|\right)$ of the first variable $z_{f}$, defined in the equations below. The magnitude of the argument of the Bessel function, say $\left|z_{f}\right|$, is really the magnitude of the corresponding oscillating energy in the interaction Hamiltonian. Here, the generalized Bessel function means the norm of a generalized phased Bessel (GPB) function. We attribute most strong-laser-field phenomena in the nonrelativistic regime to three basic dimensionless parameters: atomic binding number, ponderomotive number, and absorbed-photon number. With these parameters we found a scaling law for PADs [16].

In this paper, we continue using NPQED theory to treat 
the anomaly observed by Reichle et al. The theoretical analysis for this work is based upon the work of Gao, Guo, and $\mathrm{Wu}$ [15] from the nonperturbative scattering theory of Guo, Åberg, and Crasemann [14] with the inclusion of spontaneously emitted photon modes. The transition formula for the angular distribution of a given ATI peak is given by [15]

$$
\begin{aligned}
\left.\frac{d^{4} W}{d^{2} \Omega_{p_{f}} d^{2} \Omega_{k}}\right|_{j}= & \frac{e^{2} \omega^{9 / 2}}{\left(2 m_{e}\right)^{1 / 2}(2 \pi)^{5}}\left(j-\epsilon_{b}-u_{p}\right)^{1 / 2}\left(j-u_{p}\right)^{2} \\
& \times \sum_{q}\left(u_{p}-j+q\right)\left|\Phi_{i}\left(\mathbf{P}_{f}-q \mathbf{k}+\mathbf{k}^{\prime}\right)\right|^{2} \\
& \times\left|\mathcal{X}_{q}\left(\mathbf{P}_{f}, \mathbf{k}^{\prime}\right)\right|^{2},
\end{aligned}
$$

where $d^{2} \Omega_{p_{f}}=\sin \theta_{f} d \theta_{f} d \phi_{f}$ is the solid angle in the momentum space of the final electron, in which $\theta_{f}$ is the scattering angle and $\phi_{f}$ is the azimuthal angle; $\epsilon_{b}$ is the atomic binding energy in units of the laser photon energy; $u_{p}=U_{p} / \omega$ is the ponderomotive number; $\mathcal{X}_{q}\left(\mathbf{P}_{f}, \mathbf{k}^{\prime}\right)$ is defined by

$$
\begin{aligned}
\mathcal{X}_{q}\left(\mathbf{P}_{f}, \mathbf{k}^{\prime}\right) \equiv & \mathcal{X}_{-j}(z, \eta) \frac{1}{\omega} \sum_{j^{\prime}} \frac{1}{u_{p}-j^{\prime}} \mathcal{X}_{-j^{\prime}}\left(z_{f}, \eta\right) \\
& \times\left\{-\left[\mathbf{P}_{f}+\left(j-q-u_{p}\right) \mathbf{k}\right] \cdot \epsilon^{\prime *} X_{q-j+j^{\prime}}\left(z_{\mathbf{k}^{\prime}}\right)\right. \\
& +e \Lambda \epsilon^{*} \cdot \epsilon^{\prime *} X_{q-j+j^{\prime}+1}\left(z_{\mathbf{k}^{\prime}}\right) \\
& \left.+e \Lambda \epsilon \epsilon^{\prime *} X_{q-j+j^{\prime}-1}\left(z_{\mathbf{k}^{\prime}}\right)\right\}
\end{aligned}
$$

and the GPB function

$$
\mathcal{X}_{j}(z, \eta) \equiv \sum_{s=-\infty}^{\infty} X_{j-2 s}(z) X_{s}(\eta)
$$

is defined in terms of phased Bessel functions $X_{n}(z)$ of a complex variable $z$ related to the ordinary Bessel functions $J_{n}(r)$ by

$$
X_{n}(z)=J_{n}(r) e^{i n \varphi} \quad \text { with } z=r e^{i \varphi} .
$$

The arguments in Eq. (2) are defined as follows:

$$
\begin{gathered}
z_{f}=2 \frac{|e| \Lambda}{m_{e} \omega} \mathbf{P}_{f} \cdot \epsilon, \quad z_{\mathbf{k}^{\prime}}=2 \frac{|e| \Lambda}{m_{e} \omega} \mathbf{k}^{\prime} \cdot \boldsymbol{\epsilon}, \\
z=z_{f}+z_{\mathbf{k}^{\prime}}, \quad \eta=\frac{1}{2} u_{p} \cos \xi,
\end{gathered}
$$

with $2 \Lambda$ being the classical amplitude of the field and $m_{e}$ is the electron mass. The $\mathbf{P}_{f}$ is the momentum of the photoelectron outside of the laser field; $\omega$ and $\mathbf{k}$ are, respectively, the frequency and wave vector of the laser light with polarization vector $\epsilon$ and polarization degree $\xi ; \mathbf{k}^{\prime}$ is the propagation vector of the spontaneous emission mode with frequency $\omega^{\prime}=\left[u_{p}-(j-q)\right] \omega$ and polarization vector $\epsilon^{\prime}$. In the expression of Eq. (2), $j$ is the transferred-photon number. Because the kinetic energy of the photoelectron in the laser field is approximately the kinetic energy of the photoelectron out of the laser field, $j$ also denotes the order of the abovethreshold-ionization (ATI) or EPD peaks. The momentum wave function $\Phi_{i}\left(\mathbf{P}_{f}-q \mathbf{k}+\mathbf{k}^{\prime}\right)$ is the Fourier transform of the initial wave function. In the Nandor et al. observation [18], the electrons emitted along the laser polarization vector form the main lobe of PADs, while jets were the prominent electrons emitted from the waist between the main lobes. In the Reichle et al. observation, the jet is the central maximal in the detected angular distributions, shown in Fig. 5 of Ref. [3]. We have shown that the jets in PADs are caused by the maxima of the GPB function and the PADs of an even-order ATI peak always have a central jet, while those of an oddorder peak have no central jet. Our results on central jets agree with earlier discussion made by Gribakin and Kuchiev [20]. The Reichle et al. observation can still confirm our statements.

The PAD for a given ATI (or EPD) peak can be obtained from Eq. (1) by integrating over the solid angle of spontaneous emission mode and summing over all possible spontaneous emission modes:

$$
\begin{aligned}
\left.\frac{d^{2} W}{d^{2} \Omega_{p_{f}}}\right|_{j}= & \frac{e^{2} \omega^{9 / 2}}{\left(2 m_{e}\right)^{1 / 2}(2 \pi)^{5}}\left(j-\epsilon_{b}-u_{p}\right)^{1 / 2}\left(j-u_{p}\right)^{2} \\
& \times \sum_{q}\left(u_{p}-j+q\right) \int d^{2} \Omega_{\mathbf{k}^{\prime}}\left|\Phi_{i}\left(\mathbf{P}_{f}-q \mathbf{k}+\mathbf{k}^{\prime}\right)\right|^{2} \\
& \times\left|\mathcal{X}_{q}\left(\mathbf{P}_{f}, \mathbf{k}^{\prime}\right)\right|^{2}
\end{aligned}
$$

In the long-wavelength approximation, the PAD, which is mainly determined by the generalized Bessel function, depends on the order $j$ of generalized Bessel function, the ponderomotive parameter $u_{p}$, and the binding number $\epsilon_{b}$ [17] and obeys a scaling law [16]. PADs are determined by the transition amplitude of photon states represented by the generalized Bessel function in the transition formula. The order of the generalized Bessel function denotes the number of absorbed photons. Here the generalized Bessel function is the leading one in Eq. (2) which is a single factor next to the sum. The factors in the sum do not affect the PADs significantly since they are summed over. It is found that the jets in PADs are due to the maxima of the GPB function. The total number of jets on one side of the PADs is determined by the number rule. Here the maximum at zero for the even-order generalized Bessel functions is counted as the halfmaximum. The even order and odd order GPBs possess even and odd parity properties, respectively. They participate the parity conservations in multiphoton transition processes.

In Fig. 5 of Ref. [3], for the two-photon EPD peak, more photoelectrons were emitted in the directions perpendicular to the polarization vector, say around emission angle $\theta$ $=90^{\circ}$ and $270^{\circ}$, than those in the poles of the polarization vector. This means that the PAD of the two-photon EPD peak has a large central jet. There is no central jet in the threephoton EPD peak. In our theory, this central jet is due to the half-side maximum of the phased Bessel function $\mathcal{X}_{-2}(z, \eta)$ at the beginning point of argument $|z|$. When $z=0$ for a fixed $\eta$, since $X_{n}(0)=\delta_{n 0}$, Eq. (3) leads to $\mathcal{X}_{-j}(0, \eta)=X_{-j / 2}(\eta)$ for even $j$. The value of $\mathcal{X}_{-j}(0, \eta)$ does not vanish only when $j$ $=$ even. We immediately arrive at the conclusion: The PAD for any even-order ATI (or EPD) peaks has a central jet, for any odd-order ones there is no central jet. In their measurement, the central jet in the four-photon EPD peak is not outstanding due to the weakness of the total rate of that peak. 

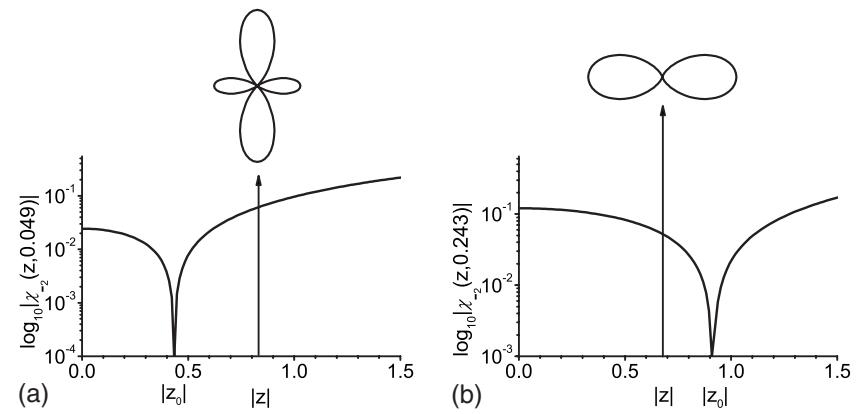

FIG. 1. Schematic diagrams of the second-order generalized Bessel function as a function of the variable $z$ for two laser intensities: (a) $I=0.13 T W / \mathrm{cm}^{2}$ and (b) $I=0.65 T W / \mathrm{cm}^{2}$. The insets are the calculated PADs with value $|z|$ indicated by the arrows. The calculation parameters are chosen as $\lambda=2150 \mathrm{~nm}$ and $E_{b}$ $=0.7521 \mathrm{eV}$.

Now we turn back to the PADs of the two-photon EPD peak shown in Fig. 5 of Ref. [3]. The observed PAD, in Fig. 5(a) of Ref. [3], are formed by two main lobes and a jet sticking out from the waist between the two main lobes. The height of the jet is lower than that of the main lobes at a lower laser intensity, $0.13 T W / \mathrm{cm}^{2}$. In Fig. 5(b) of Ref. [3], at a higher intensity $0.65 T W / \mathrm{cm}^{2}$, the main lobe disappears. Thus there only exists a large central jet vertical to the polarization vector. The explanation given by Ref. [3] is the interference effect between the ionization channels leading to $s$ and $d$ waves. To this phenomenon, our explanation is the following: the large central jet signifies the much larger value of $\mathcal{X}_{-2}(0, \eta)$ than that of $\mathcal{X}_{-2}\left(z_{f}, \eta\right)$ and the function $\mathcal{X}_{-2}(z, \eta)$, as the function of $z$, has no other maximum or half-side maximum in the domain of $\left[0,\left|z_{f}\right|\right]$. The maximum we mean here is that of the absolute value of the generalized Bessel functions.

In the following, we are going to show how $\left|z_{f}\right|$ is determined by the final kinetic energy of a photoelectron through the Einstein relation (the energy conservation) in the multiphoton case with the inclusion of the ponderomotive energy. The disappearance of the main lobes are due to the decrease of $z$ argument with an increasing field intensity for a given ATI (or EPD) peak. According to Ref. [15], the derived Einstein relation gives the final kinetic energy of the photoelectron as

$$
\frac{\mathbf{p}_{f}^{2}}{2 m_{e}} \approx j \omega-u_{p} \omega-E_{b}
$$

where $j$ is the number of photons absorbed in the ionization process, $u_{p} \omega$ is the ponderomotive energy. For the twophoton EPD peak produced from the target $\mathrm{H}^{-}$ions, in the current discussion, $j=2$ and $E_{b}=0.7521 \mathrm{eV}$ are fixed parameters; while the final kinetic energy of the photoelectron decreases linearly with the increasing laser intensity through the increasing ponderomotive energy, that is often the ponderomotive shift. This shift reduces the final kinetic energy, as well as the final momentum, of the photoelectron; and in turn reduces the $z_{f}$ argument of the phased Bessel function. With Eq. (6), this dependence can be shown as follows:

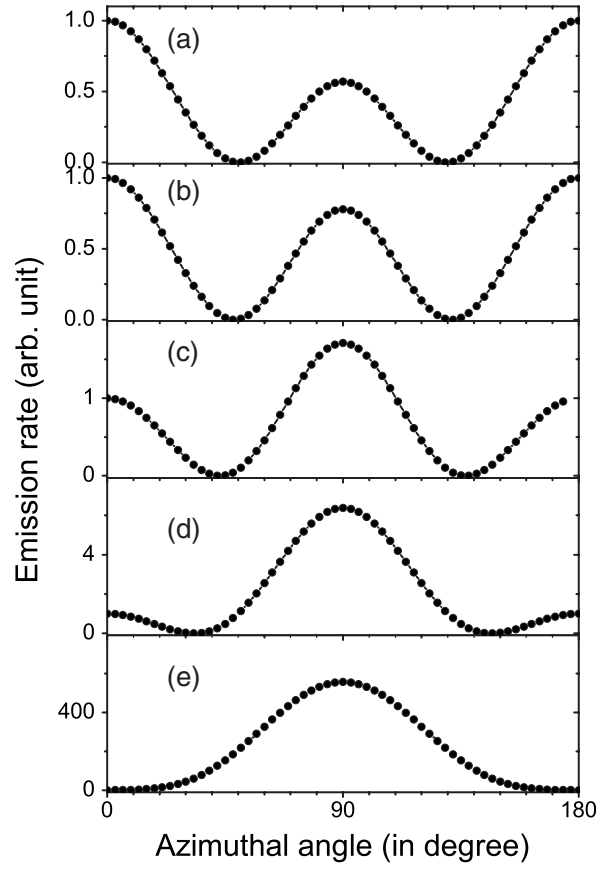

FIG. 2. The calculated PADs for two-photon EPD peak for $H^{-}$at different laser intensties: (a) $I=0.13 T W / \mathrm{cm}^{2}$; (b) $I=0.2 T W / \mathrm{cm}^{2}$; (c) $I=0.35 T W / \mathrm{cm}^{2}$; (d) $I=0.50 T W / \mathrm{cm}^{2}$; and (e) $I=0.65 T W / \mathrm{cm}^{2}$. Other parameters are the same as those in Fig. 1.

$$
z_{f} \equiv \frac{2|e| \Lambda}{m_{e} \omega} \mathbf{P}_{f} \cdot \epsilon=2 \sqrt{2 u_{p}\left(j-u_{p}-\epsilon_{b}\right)} \cos \phi=\left|z_{f}\right| \cos \phi,
$$

where $\left|z_{f}\right|=2 \sqrt{2 u_{p}\left(j-u_{p}-\epsilon_{b}\right)}$ and $\phi=\hat{\mathbf{P}}_{f} \cdot \boldsymbol{\epsilon}$ is the azimuthal angle, which was labeled as $\theta$ in Ref. [3]. The $\eta$ argument increases linearly with increasing laser intensity for a fixed laser polarization. This increase does not alter qualitatively the geometric characters of the GPB function $\mathcal{X}_{j}(z, \eta)$, as a function of its first argument $z$. For simplicity, we ignore those nonqualitative changes.

The calculated PADs using our formula, with the Reichle et al. experimental parameters as the input, are presented in Figs. 1(a) and 1(b) which correspond to Figs. 5(a) and 5(b) of Ref. [3], respectively. The relation between PADs and geometric shape of the GPB functions are showed in our plots. In case (a), the laser intensity is low enough, thus the domain $\left[0,|z|_{f}\right]$ is large enough to cover the only zero point $|z|_{0}$, which is the only sharp minimum of the GPB function. The interval $\left[0,\left|z_{0}\right|\right]$ gives the central jet, while the $\left[|z|_{0},\left|z_{f}\right|\right]$ gives the main lobes. In case (b), in the higher intensity, the domain $\left[0,|z|_{f}\right]$ is not large enough to cover the only zero point $|z|_{0}$ due to the small value of $\left|z_{f}\right|$. In the interval $\left[0,|z|_{f}\right] \subseteq\left[0,|z|_{0}\right]$ photoelectrons can form only the central jet only with vanishing main lobes. This is the reason for the disappearance of the main lobes. With further higher laser intensities, the only central jet can vanish, that is channel suppression due to the further increased ponderomotive energy added to the threshold energy in the Einstein relation.

To see clearly how the main lobes gradually vanish with an increasing laser intensity for a given ATI peak, we refine the calculations between case (a) and case (b). These calcu- 


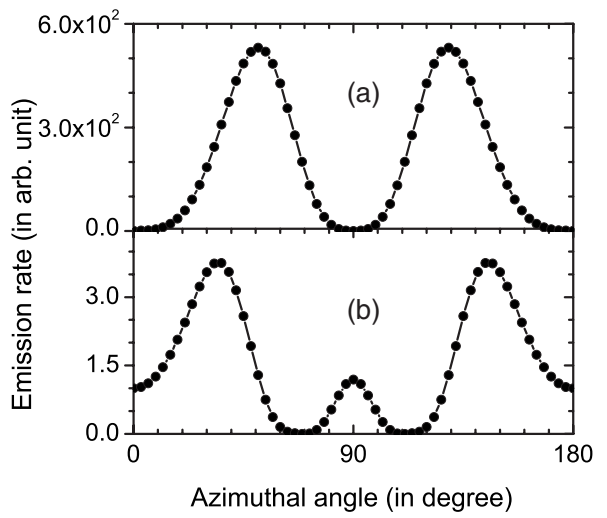

FIG. 3. The PADs of the first EPD peak for $H^{-}$at different laser intensties: (a) $I=1.5 T W / \mathrm{cm}^{2}$ (three-photon EPD) and (b) $I$ $=2.5 T \mathrm{~W} / \mathrm{cm}^{2}$ (four-photon EPD). Other parameters are the same as those in Fig. 1.

lations show that our theory not only predicts the isolated PADS in different laser intensities, but also predicts all PAD formation processes with continuous variation of intermediate laser intensities (Fig. 2)

For further larger $\left|z_{f}\right|$, which occurs at higher-order ATI (or EPD) peaks at relatively low intensities, the main lobes may have hierarchy disappearances. These hierarchy anomalies are calculated in Figs. 3(a) and 3(b). As a PAD of a three-photon EPD process, Fig. 3(a) shows a new disappearance of main lobes. Since there is no central jet in the oddphoton case, the plot shows a pair of dominating side jets. As a PAD of a four-photon EPD process, Fig. 3(b) shows another disappearance of the main lobes. Since there is central jet in the even-photon case, the plot shows a pair of large side jets in addition to the small central jet.

In summary, the partial-wave mixing scheme using Legendre polynomials to fit PADs can account for parity conservation well, but cannot determine the artificial parameter theoretically. The parameter was determined by other sources. Our scheme, the Bessel function scheme, has the following advantages: (1) It can account for the parity conservation. (2) It has no fitting parameter. (3) It can predict the formation processes of all different PADs. (4) It can also predict hierarchy disappearance of the main lobes. We hope that in the near future our predictions can be verified by experiments. Using generalized Bessel functions to describe electron transition amplitude due to absorbing and emitting photons is a common feature of most nonperturbative theories. We would like to see that other nonperturbative theories, such as Keldysh-Faisal-Reiss (KFR) [19] theory, may also lead to the similar conclusions that we made. The partialwave scheme is more appropriate to perturbative theories which treat transferred photons one by one with changing the angular quantum number also one by one. While, the Bessel function scheme is more appropriate to nonperturbative theories where the transferred photon numbers are directly denoted by the order of Bessel functions or generalized Bessel functions.

One of the authors (J.Z.) is supported by the rising star programme of Shanghai and the Chinese National Natural Science Foundation under Grant No. 60408008 and the 973 program of China. One of the authors (D.-S.G.) is supported by the summer grant of the Physics Department of Ohio State University.
[1] R. R. Freeman, Phys. World 5, 29 (1992).

[2] I. Y. Kiyan and H. Helm, Phys. Rev. Lett. 90, 183001 (2003).

[3] R. Reichle, H. Helm, and I. Y. Kiyan, Phys. Rev. Lett. 87, 243001 (2001).

[4] D. A. Telnov and Shih-I Chu, Phys. Rev. A 66, 063409 (2002); 66, 043417 (2002).

[5] R. Reichle, H. Helm, and I. Y. Kiyan, Phys. Rev. A 68, 063404 (2003).

[6] J. R. Taylor, Scattering Theory: The Quantum Theory of Nonrelativistic Collisions (Robert E. Krieger, Marlabar, FL, 1983), pp. 180-237.

[7] C. N. Yang, Phys. Rev. 74, 764 (1948).

[8] J. Cooper and R. N. Zare, in Lectures in Theoretical Physics: Atomic Collision Processes, edited by S. Geltman, K. T. Mahanthappa, and W. E. Britten (Gordon and Breach, New York, 1969), Vol. XI-C, pp. 317-337.

[9] S. T. Manson and A. F. Starace, Rev. Mod. Phys. 54, 389 (1982).

[10] K. G. Kim, C. C. Widmayer, and M. D. Girardeau, Phys. Rev. A 47, 2856 (1993).

[11] M. Bashkansky, P. H. Bucksbaum, and D. W. Schumacher,
Phys. Rev. Lett. 60, 2458 (1988).

[12] R. R. Freeman, P. H. Bucksbaum, H. Milchberg, S. Darack, D. Schumacher, and M. E. Geusic, Phys. Rev. Lett. 59, 1092 (1987).

[13] P. Hansch, M. A. Walker, and L. D. Van Woerkom, Phys. Rev. A 57, R709 (1998).

[14] D.-S. Guo, T. Aberg, and B. Crasemann, Phys. Rev. A 40, 4997 (1990).

[15] J. Gao, D.-S. Guo, and Y.-S. Wu, Phys. Rev. A 61, 043406 (2000).

[16] D.-S. Guo, J. Zhang, Z. Xu, X. Li, P. Fu, and R. R. Freeman, Phys. Rev. A 68, 043404 (2003).

[17] J. Zhang, W. Zhang, Z. Xu, X. Li, P. Fu, D.-S. Guo, and R. R. Freeman, J. Phys. B 35, 4809 (2002).

[18] M. J. Nandor, M. A. Walker, and L. D. van Woerkom, J. Phys. B 31, 4617 (1998).

[19] L. V. Keldysh, Zh. Eksp. Teor. Fiz. 47, 1945 (1964) [Sov. Phys. JETP 20, 1307 (1965)]; F. H. M. Faisal, J. Phys. B 6, L89 (1973); H. R. Reiss, Phys. Rev. A 22, 1786 (1980).

[20] G. F. Gribakin and M. Y. Kuchiev, Phys. Rev. A 55, 3760 (1997). 